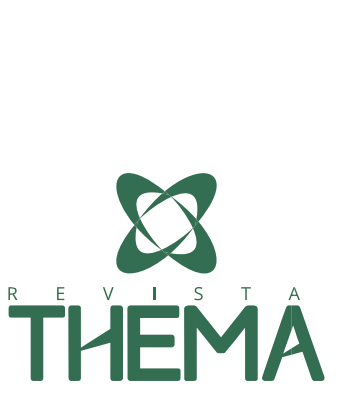

\title{
Estudo Numérico da Frequência de Oscilação de dois Pêndulos Acoplados por uma Mola
}

\author{
Numerical Study of the Frequency of Oscillation of Two \\ Pendulums Coupled by a Spring
}

Octávio José Santos Santana ${ }^{1}$; Romualdo Santos Silva Jr. ${ }^{2}$

\section{RESUMO}

Neste trabalho estudamos numericamente as frequências de oscilação de dois pêndulos acoplados por uma mola, considerando que os mesmos apresentam massas diferentes. Através das equações de movimento, obtemos numericamente os pontos fixos do sistema, os quais representam pontos no espaço de fase em que o sistema permanece estacionário. Escolhemos algumas condições iniciais do problema próximo de um ponto fixo especifico, e analisamos numericamente as frequências de oscilações de cada pêndulo no regime de pequenas oscilações e fora do regime de pequenas oscilações. Confirmamos que no regime de pequenas oscilações, os modos de vibrações dos pêndulos oscilam em fase e oposição de fase, como também em que situação este regime não é válido. Além disso, considerando que os pêndulos oscilam com fases opostas, verifica-se que o sistema apresenta comportamento caótico.

Palavras-chave: Pontos fixos; Frequência de oscilação; Pêndulos acoplados.

\begin{abstract}
In this work we study numerically the oscillation frequencies of two pendulums coupled by a spring, considering that they have different masses. Through the equations of motion, we obtain numerically the fixed points of the system, which represent points in the phase space in which the system remains stationary. We chose some initial conditions of the problem close to a specific fixed point, and numerically analyzed the frequencies of oscillations of each pendulum in the regime of small oscillations and outside the regime of small oscillations. We confirm that in the regime of small oscillations, the vibration modes of the pendulums oscillate in phase and phase opposition, as well as in what situation this regime is not valid. In addition, considering that the pendulums oscillate with opposite phases, it is verified that the system presents chaotic behavior.
\end{abstract}

Keywords: Fixed points; Oscillation frequency; Coupled pendulums.

\footnotetext{
${ }^{1}$ UNICAMP - Universidade Estadual de Campinas, Campinas/SP - Brasil

2 UFS - Universidade Federal de Sergipe, São Cristóvão/SE - Brasil
} 


\section{INTRODUÇÃO}

É sabido que as oscilações desempenham um papel fundamental na física, seja ela na mecânica, na acústica, na eletricidade, como também na ótica. Um dos sistemas mais simples no que diz respeito ao estudo de oscilações harmônicas é o sistema massa-mola, que é caracterizado por um corpo (massa), acoplado a outro corpo material (mola), mantido em sua posição de equilíbrio, onde a mola se encontra sem deformações, sendo assim, portanto, livre de tensões internas. Quando realizamos o deslocamento de sua posição de equilíbrio, a massa sofre a ação de uma força restauradora linear, e esta tende a retornar ao ponto de equilíbrio. Esta força é devida à tendência da mola de retomar ao seu estado original, sem sofrer nenhum tipo de deformações como também tensões internas.

Outro sistema interessante que tem sido estudado até hoje é o pêndulo físico, citamos como exemplos o pêndulo duplo, pêndulo invertido e pêndulo acoplado. Um sistema bastante trivial que se pode estudar é o pêndulo simples, onde o mesmo é encontrado em diversos livros de física básica (GOLDSTEIN, 2000; LANDAU, 1996), métodos matemáticos de física (ARFKEN, 1985; BENDER, 1978), métodos computacionais e textos específicos de oscilações (CAP, 2003; BILLINGHAM, 2000; PAIN, 2005).

Quando consideramos dois pêndulos com massas $(\mathrm{m})$ e comprimentos $(\mathrm{L})$ iguais acoplados por uma mola de constante elástica ( $k$ ), como mostra a Figura 1, já é sabido que seus modos normais de vibração são descritos por duas frequências de oscilação, em que a primeira $\left(\omega_{1}\right)$ está relacionado com o comprimento do pêndulo e a segunda $\left(\omega_{2}\right)$ com a massa, tal que, se $\omega=\omega_{1}$, os pêndulos oscilam em fase, entretanto, se $\omega=\omega_{2}$ os pêndulos oscilam em fase oposta. Além disso, o estudo de corpos oscilatórios com acoplamento entre eles podem levar muitas vezes à fenômenos interessantes, caracterizados pela sincronização e pela diferença de fase entre eles (AVILA, 2003).

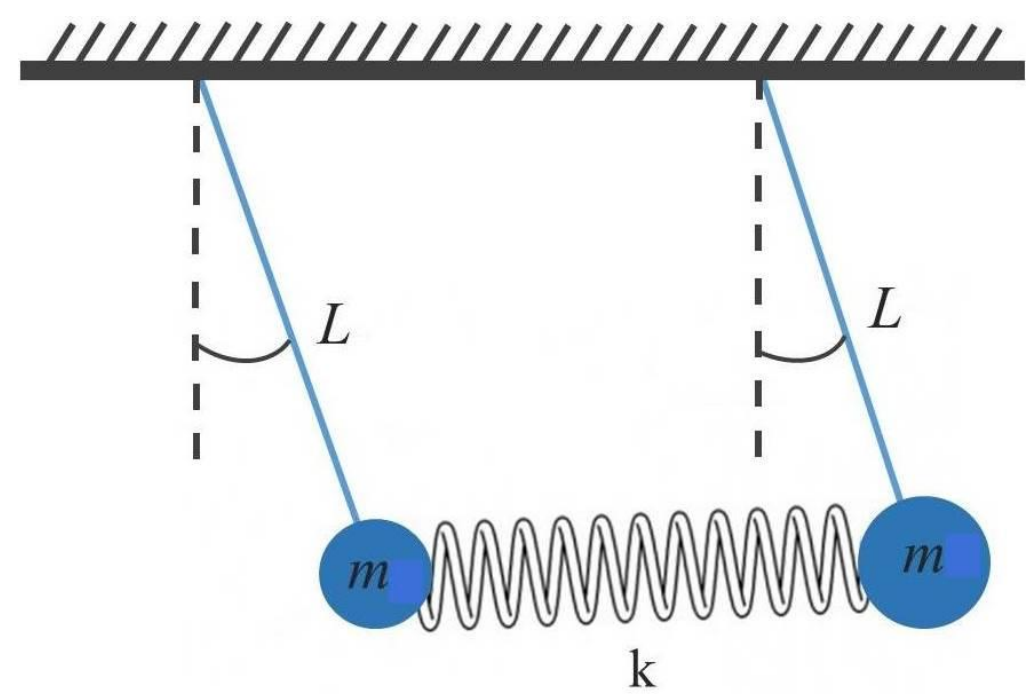

Figura 1: Esquema de dois pêndulos com massas $m$ e comprimentos $L$ acoplados por uma mola de constante elástica $k$ Fonte: autor

Em um trabalho recente publicado Silva Jr. (2013), foi proposto um sistema que consiste de dois pêndulos com massas diferentes acoplados por uma mola, onde o parâmetro $m_{a, b}$ é a massa de cada pêndulo, $L_{a}=L_{b}$ é o comprimento da cada pêndulo e $k$ a constante elástica da mola, como mostra a Figura 2. Neste trabalho, foi observado que a segunda frequência de oscilação agora se comporta em função da massa de cada pêndulo, mais especificamente da soma e do produto entre elas. 


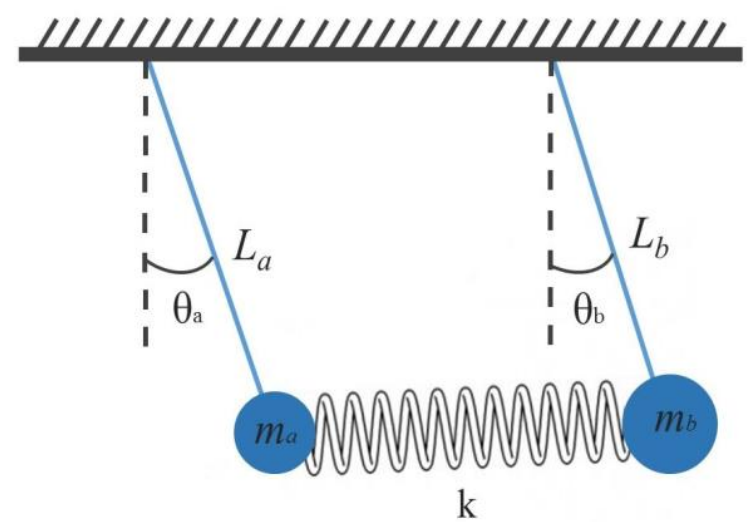

Figura 2: Esquema do Sistema Físico proposto

Fonte: autor

Tomando como referência o problema proposto anteriormente (SILVA JR, 2013), objetiva-se estender este trabalho, através de um estudo numérico da estabilidade e frequências de oscilação dos pêndulos acoplados de maneira geral.

\section{MODELO TEÓRICO}

A Hamiltoniana que descreve o sistema proposto é escrita como:

$$
\begin{gathered}
H=\frac{P_{\theta_{a}}^{2}}{2 m_{a} L_{a}^{2}}+\frac{P_{\theta_{b}}^{2}}{2 m_{b} L_{b}^{2}}-g m_{a} L_{a} \cos \left(\theta_{a}\right)-g m_{b} L_{b} \cos \left(\theta_{b}\right)+\frac{1}{2} k L_{a}^{2} \sin ^{2}\left(\theta_{a}\right)+\frac{1}{2} k L_{b}^{2} \sin ^{2}\left(\theta_{b}\right) \\
-k L_{a} \sin \left(\theta_{a}\right) L_{b} \sin \left(\theta_{b}\right),
\end{gathered}
$$

sendo $k$ a constante elástica da mola, $g$ a constante gravitacional, $m_{a}$ e $m_{b}$ as massas, $L_{a}$ e $L_{b}$ os comprimentos, $\theta_{a}$ e $\theta_{b}$ as coordenadas angulares, e $P_{\theta_{a}}$ e $P_{\theta_{b}}$ os momentos angulares dos pêndulos $a$ e $b$, respectivamente.

Desta forma, conhecendo o sistema e a hamiltoniana que descreve o mesmo, podemos escrever usando o formalismo hamiltoniano as equações na evolução temporal, sendo então:

$$
\frac{d q_{i}}{d t}=\frac{\partial H}{\partial p} \quad \text { e } \quad \frac{d p_{i}}{d t}=-\frac{\partial H}{\partial q}
$$

Assim, as equações que descrevem a dinâmica deste sistema são escritas como:

$$
\begin{gathered}
\frac{d \theta_{a}}{d t}=\frac{P_{\theta_{a}}}{m_{a} L_{a}^{2}} \\
\frac{d \theta_{b}}{d t}=\frac{P_{\theta_{b}}}{m_{b} L_{b}^{2}} \\
\frac{d P_{\theta_{a}}}{d t}=-g m_{a} L_{a} \sin \left(\theta_{a}\right)-k L_{a}^{2} \sin \left(\theta_{a}\right) \cos \left(\theta_{a}\right)+k L_{a} L_{b} \cos \left(\theta_{a}\right) \sin \left(\theta_{b}\right) \\
\frac{d P_{\theta_{b}}}{d t}=-g m_{b} L_{b} \sin \left(\theta_{b}\right)-k L_{b}^{2} \sin \left(\theta_{b}\right) \cos \left(\theta_{b}\right)+k L_{b} L_{a} \cos \left(\theta_{b}\right) \sin \left(\theta_{a}\right)
\end{gathered}
$$


Foi escrito um código em Python versão 2.7 para realizar os cálculos numéricos, onde utilizamos o método de Runge Kutta de quarta ordem (STROGATZ, 1998) para obter as soluções das equações diferenciais.

\section{RESULTADOS E DISCUSSÕES}

Inicialmente vamos analisar os pontos fixos do sistema e sua estabilidade. Pontos fixos são pontos representados no espaço de fase no qual a taxa de variação temporal é nula. No nosso modelo, notase que nas equações (3) e (4) os pontos fixos são $P_{\theta_{a}}^{*}=P_{\theta_{b}}^{*}=0$ como pode ser visto nas equações (7) e (8), enquanto as equações (5) e (6) apresenta muitos pontos no espaço de fase em que a taxa de variação temporal dos momentos é nula.

$$
\begin{gathered}
\frac{d \theta_{a}}{d t}=0 \Rightarrow \frac{P_{\theta_{a}}^{*}}{m_{a} L_{a}^{2}}=0 \Rightarrow P_{\theta_{a}}^{*}=0 \\
\frac{d \theta_{b}}{d t}=0 \Rightarrow \frac{P_{\theta_{b}}^{*}}{m_{b} L_{b}^{2}}=0 \Rightarrow P_{\theta_{b}}^{*}=0 \\
\frac{d P_{\theta_{a}}}{d t}=0 \Rightarrow-g m_{a} L_{a} \sin \left(\theta_{a}^{*}\right)-k L_{a}^{2} \sin \left(\theta_{a}^{*}\right) \cos \left(\theta_{a}^{*}\right)+k L_{a} L_{b} \cos \left(\theta_{a}^{*}\right) \sin \left(\theta_{b}^{*}\right)=0 \\
\frac{d P_{\theta_{b}}}{d t}=0 \Rightarrow-g m_{b} L_{b} \sin \left(\theta_{b}^{*}\right)-k L_{b}^{2} \sin \left(\theta_{b}^{*}\right) \cos \left(\theta_{b}^{*}\right)+k L_{b} L_{a} \cos \left(\theta_{b}^{*}\right) \sin \left(\theta_{a}^{*}\right)=0
\end{gathered}
$$

As equações (9) e (10) representam a taxa de variação temporal dos momentos nula, e por serem equações transcendentais não apresenta solução analítica, de modo que sua solução é obtida numericamente.

A Figura 3 mostra o espaço de fase das coordenadas angulares. As regiões em azul, vermelho e preto correspondem à taxa de variação temporal ser positiva, negativa e nula, respectivamente.
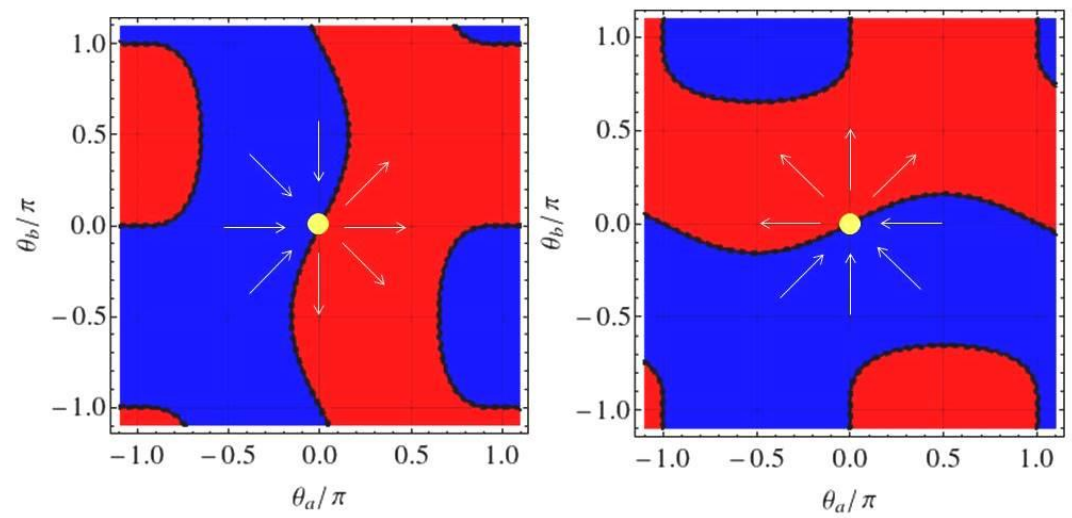

Figura 3: Taxa de variação dos momentos lineares em funções das coordenadas angulares, para o pêndulo ' $a$ ' e ' $b$ ', respectivamente. Foram utilizados os seguintes parâmetros: $\mathrm{m}_{\mathrm{a}}=\mathrm{m}_{\mathrm{b}}=1.0 \mathrm{~kg}, \mathrm{~L}_{\mathrm{a}}=\mathrm{L}_{\mathrm{b}}=1.0 \mathrm{~m}, \mathrm{k}=10.0 \mathrm{~N} / \mathrm{m}$ e g $=9.8 \mathrm{~m} / \mathrm{s}^{2}$.

Fonte: GOLDSTEIN, 2000

Pode-se analisar a dinâmica do sistema através de campos vetoriais, onde o sinal da taxa de variação temporal informa em que direção o campo vetorial "flui" (representado pelas setas, com relação a um dos pontos fixos do sistema). Ou seja, a região em preto é denominada de pontos fixos e significa regiões onde as soluções são estacionárias (não varia com o tempo), enquanto a região em azul são 
regiões no qual o fluxo dos campos vetoriais se aproxima dos pontos fixos, e por fim as regiões em vermelho representa a situação em que o fluxo se afasta dos pontos fixos. Portanto para os pontos fixos que coincidem nos dois espaços de fase, a dinâmica do sistema será estacionaria, por exemplo, os pontos em amarelo da Figura 3 são pontos fixos e coincidem nos dois espaços de fase. Eles correspondem a $\theta_{a}^{*}=\theta_{b}^{*}=0$, que se usada como condição inicial do problema a solução é estacionária, pois nesta situação a força resultante no sistema é nula.

A Figura 4 apresenta a evolução temporal do espaço de fase das coordenadas angulares normalizada por $\Pi$, onde desta vez variamos as massas dos pêndulos fazendo $m_{a}=1.0 \mathrm{~kg} \mathrm{e} m_{b}=2.0 \mathrm{~kg}$, e fixando $\mathrm{L}_{\mathrm{a}}=\mathrm{L}_{\mathrm{b}}=1.0 \mathrm{~m}, \mathrm{k}=10.0 \mathrm{~N} / \mathrm{m} \mathrm{e} \mathrm{g}=9.8 \mathrm{~m} / \mathrm{s}^{2}$. A curva em preto representa as condições iniciais $P_{\theta_{a}}=$ $P_{\theta_{b}}=0$ e $\theta_{a}=\theta_{b}=0,03 \pi$, e a curva em azul as condições $P_{\theta_{a}}=P_{\theta_{b}}=0$ e $\theta_{a}=-0,03 \pi$ e $\theta_{b}=$ $0,03 \pi$. Visivelmente na curva em preto, os dois pêndulos estão em fase, neste caso a mola não é nem comprimida e nem estendida, consequentemente não há transferência de energia entre os pêndulos. Para a curva em azul, os pêndulos iniciam o movimento em posições opostas, e por se encontrarem acoplados a uma mola, consequentemente a dinâmica de cada pêndulo será afetada ocorrendo transferência de energia entre eles, afetando a amplitude de oscilação de cada pêndulo.

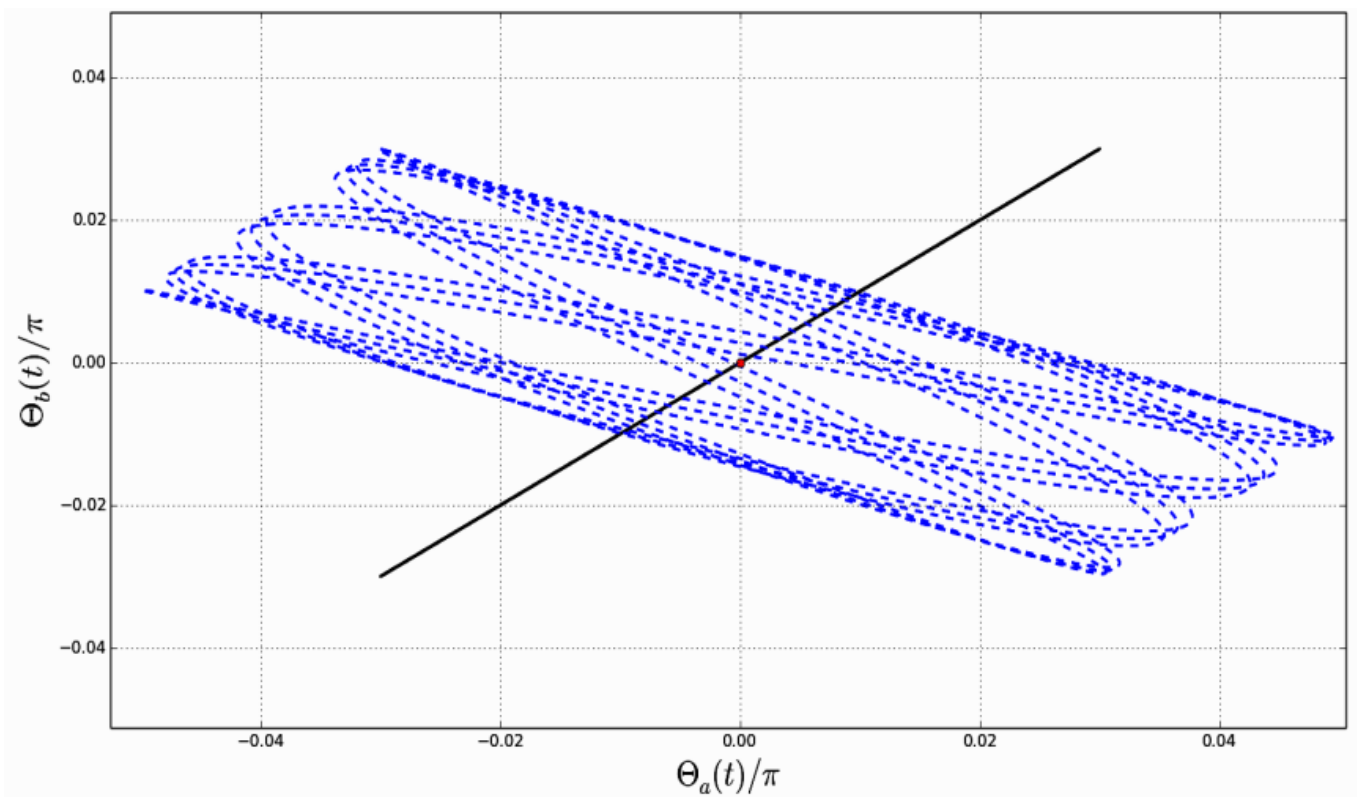

Figura 4: Espaço de fase das coordenadas angulares normalizada por $\Pi$. Com as condições inicias do problema $P_{\theta_{a}}=P_{\theta_{b}}=0$ e $\theta_{a}=\theta_{b}=0,03 \pi$, referente à curva em preto contínuo e $P_{\theta_{a}}=P_{\theta_{b}}=0$ e $\theta_{a}=-0,03 \pi$ e $\theta_{b}=0,03 \pi$ e referente à curva em azul tracejado.

As curvas apresentadas na Figura 5 representam a dinâmica temporal das coordenadas angulares normalizadas por $n$ em função do tempo 5(a) e em 5(b) refere-se a transformada de Fourier das séries temporais da figura $5(\mathrm{a})$. Foram utilizados os seguintes valores de parâmetros: $m_{a}=1.0 \mathrm{~kg}$, $\mathrm{m}_{\mathrm{b}}=2.0 \mathrm{~kg}, \mathrm{~L}_{\mathrm{a}}=\mathrm{L}_{\mathrm{b}}=1.0 \mathrm{~m}, \mathrm{k}=10.0 \mathrm{~N} / \mathrm{m}$ e g $=9.8 \mathrm{~m} / \mathrm{s} 2$, com condições iniciais $P_{\theta_{a}}=P_{\theta_{b}}=0$ e $\theta_{a}=\theta_{b}=0,03 \pi$.

Como já tínhamos mencionado, essa condição inicial do sistema representa a situação onde os pêndulos oscilam em fase, tal condição revela que a única frequência de oscilação é a frequência natural do pêndulo simples. 

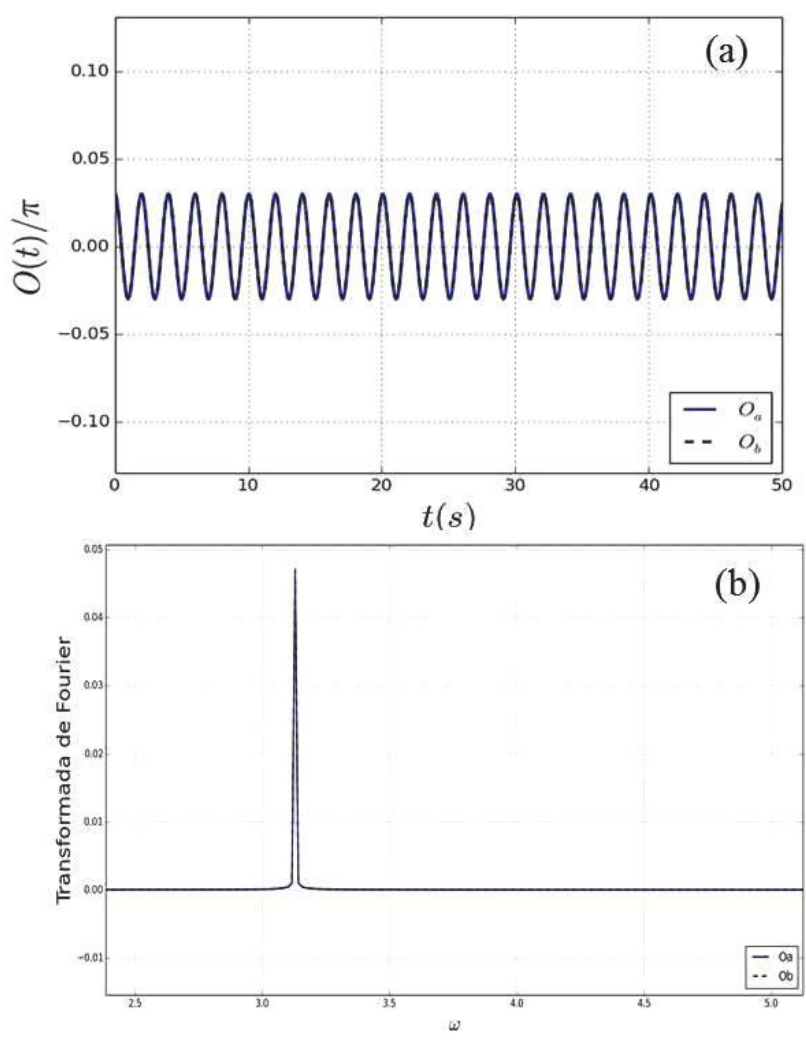

Figura 5: Coordenadas angulares normalizadas por $п$ em função do tempo (a) e a transformada de Fourier da mesma (b) Fonte: ARFKEN, 1985

Mantendo os mesmos parâmetros da Figura 5, mas alterando as condições iniciais das coordenadas angulares do sistema para $\theta_{a}=-0,03 \pi$ e $\theta_{b}=0,03 \pi$ e, observa-se que para essa configuração a dinâmica dos pêndulos é afetada devido ao acoplamento entre elas por uma mola, além de apresentar uma modulação na amplitude de oscilação de cada pêndulo. Nesta configuração a dinâmica do sistema apresenta duas frequências de oscilações, sendo que a frequência que corresponde ao acoplamento é a dominante no movimento.
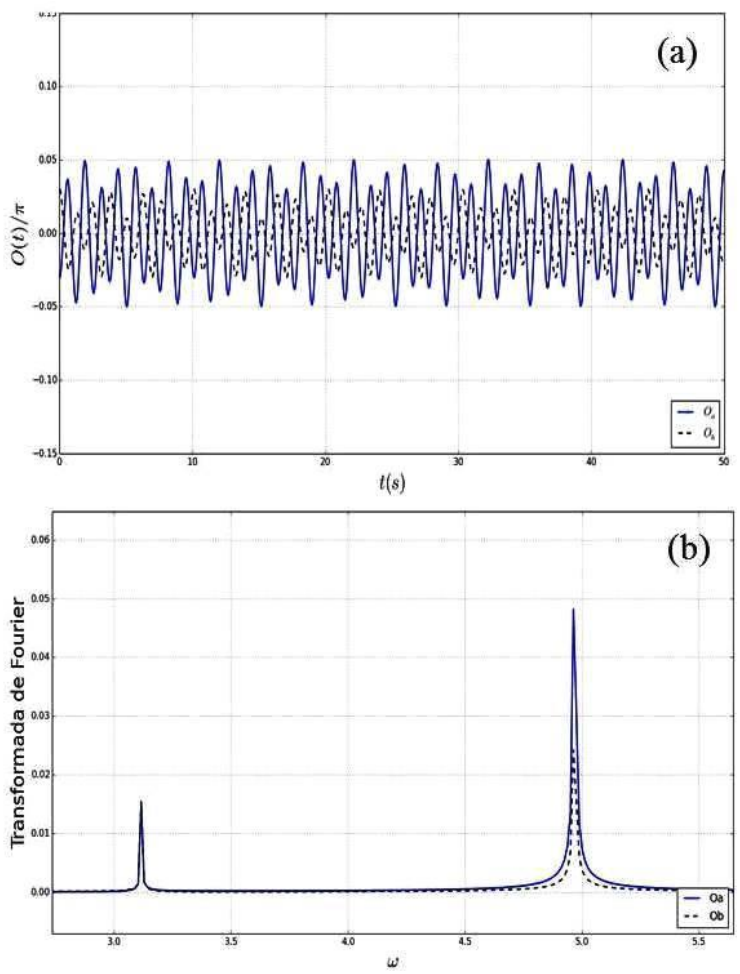

Figura 6: Coordenadas angulares normalizadas por $\Pi$ em função do tempo (a) e a transformada de Fourier da mesma (b). 
No trabalho de Silva Jr. (2013) o problema foi tratado para pequenas oscilações no qual foi possível obter facilmente os modos de vibrações do sistema. Aqui estendemos para o caso de ângulos maiores, que estariam fora do regime de pequenas oscilações. Considerando as condições iniciais $P_{a}=P_{b}=0$, $\theta_{a}=-0,3 \pi$ e $\theta_{b}=0,3 \pi$ e, verifica-se que a dinâmica do sistema entra no regime caótico, como é mostrado na figura 7(b), no qual a dinâmica do sistema apresenta várias frequências de oscilações.
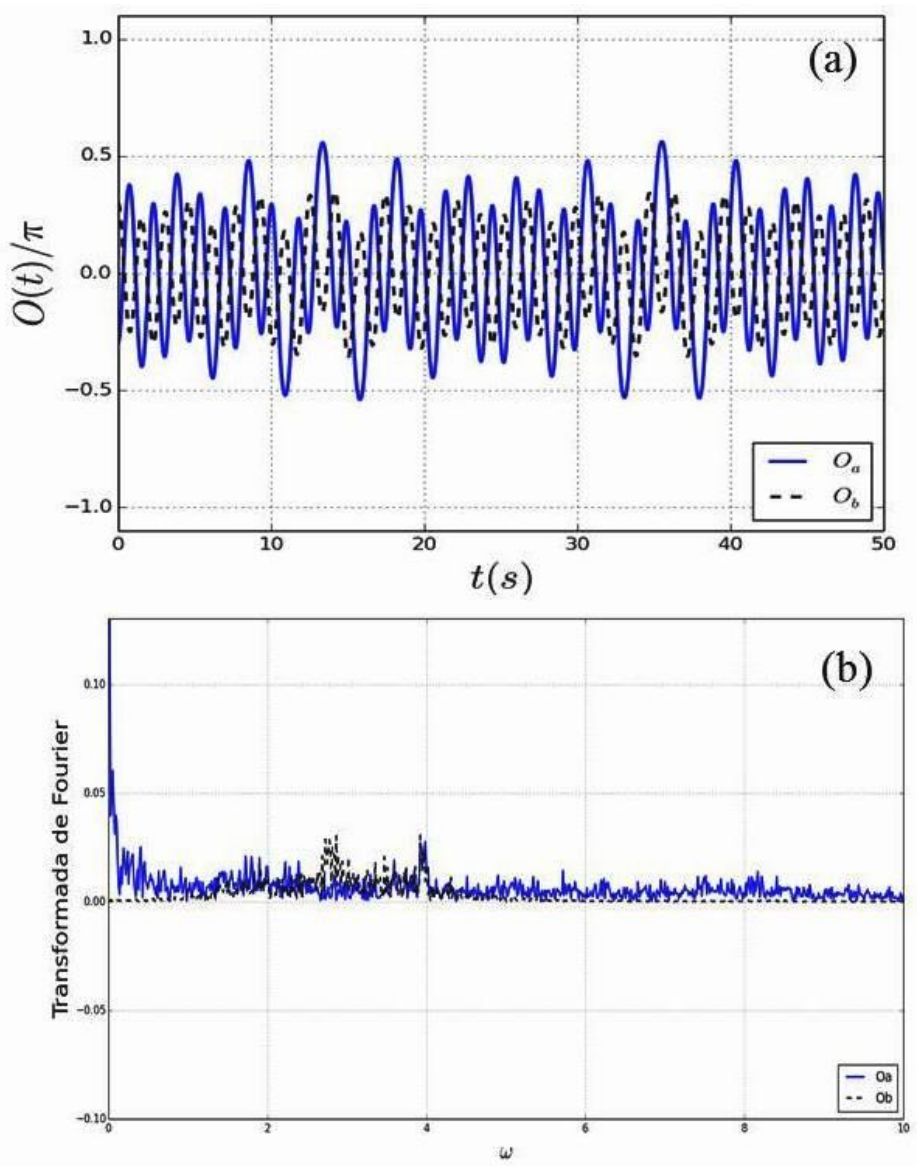

Figura 7: Coordenadas angulares normalizadas por $n$ em função do tempo (a) e a transformada de Fourier da mesma (b).

\section{CONCLUSÕES}

Através deste trabalho foi possível estudar numericamente as frequências de oscilação de dois pêndulos acoplados por uma mola, considerando que os mesmos apresentam massas diferentes. Através das equações de movimento, determinamos os pontos fixos do sistema, no qual, obtemos $P_{\theta_{a}}^{*}=P_{\theta_{b}}^{*}=0$, enquanto as coordenadas angulares apresentavam um conjunto de pontos fixos, que foi obtido numericamente e ilustrado na figura 3. Em seguida confirmamos numericamente no regime de pequenas oscilações os modos de vibrações para os pêndulos oscilando em fase e com fase opostas, além de estender a situação no qual o regime de pequenas oscilações não é válido. Neste regime e considerando os pêndulos oscilando com fases opostas verifica-se que o sistema apresenta comportamento caótico. 


\section{REFERÊNCIAS}

C. M. BENDER \& S. A. ORSZAG, Advanced mathematical methods for scientists and engineers. New York: McGraw-Hill, Inc., 1978.

F. F. CAP, Mathematical methods in physics and engineering with Mathematica. Boca aton: CRC Press, 2003.

G. ARFKEN, Mathematical methods for physicists. New York: Academic Press, Inc., 1985. G. M. RAMIREZ AVILA, J. L. GUISSET, \& J. L. DENEUBOURG, "Synchronization in light-controlled oscillators", Physica D: Nonlinear Phenomena, vol. 182, pp. 254-273, 2003.

H. GOLDSTEIN, C. POOLE, \& J. SAFKO, Classical mechanics. New York: Addison Wesley, 2000.

H. J. PAIN, The physics of vibrations and waves. Chichester: John Wiley \& Sons Ltd., 2005.

J. BILLINGHAM \& A. C. KING, Wave motion. Cambridge: Cambridge University Press, 2000.

L. LANDAU \& E. LIFCHITZ, Mecanique. Moscou: Mir, 1966.

R. S. SILVA JR.. Formalismo Hamiltoniano: Modos normais de vibração de dois pêndulos com massas diferentes acoplados por uma mola, Lat. Am. J. Phys. Educ. Vol. 7, No. 1, March 2013.

S. H. STROGATZ. Nonlinear Dynamics and Chaos. 1998. 